\title{
Medical Image Analysis of Brain X-ray CT Images By Deep GMDH-Type Neural Network
}

\author{
Tadashi Kondo ${ }^{\dagger}$ \\ Graduate School of Health Sciences, Tokushima University \\ 3-18-15 Kuramoto-cho Tokushima 770-8509 Japan \\ Email: ${ }^{\prime k}$ kondomedsci@gmail.com \\ Junji Ueno \\ Graduate School of Health Sciences, Tokushima University \\ 3-18-15 Kuramoto-cho Tokushima 770-8509 Japan \\ Shoichiro Takao \\ Graduate School of Health Sciences, Tokushima University \\ 3-18-15 Kuramoto-cho Tokushima 770-8509 Japan
}

\begin{abstract}
The deep Group Method of Data Handling (GMDH)-type neural network is applied to the medical image analysis of brain X-ray CT image. In this algorithm, the deep neural network architectures which have many hidden layers and fit the complexity of the nonlinear systems, are automatically organized using the heuristic self-organization method so as to minimize the prediction error criterion defined as Akaike's Information Criterion (AIC) or Prediction Sum of Squares (PSS). The learning algorithm is the principal component-regression analysis and the accurate and stable predicted values are obtained. The recognition results show that the deep GMDH-type neural network algorithm is useful for the medical image analysis of brain X-ray CT images.
\end{abstract}

Keywords: Deep neural networks, GMDH, Medical image recognition, Evolutionary computation, X-ray CT image.

\section{Introduction}

The deep GMDH-type neural network algorithms were proposed in our early works $^{1 \sim 4}$ and can automatically organize the neural network architectures using heuristic self-organization method $^{5,6}$ which is a type of the evolutionary computation. In this study, deep GMDH-type neural network algorithm ${ }^{1}$ is applied to the medical image analysis of brain X-ray CT images. The learning calculations of the weights is the principal component-regression analysis and the accurate and stable predicted values are obtained. In this GMDH-type neural network, two types of neurons such as the sigmoid function neuron and the polynomial neuron are used to organize the deep neural network and very complex nonlinear systems can be identified using this deep GMDH-type neural network. In our previous work $^{2}$, we have applied the deep multilayered GMDHtype neural network to the medical image recognition of brain and blood vessels. This deep multi-layered GMDH-type neural network selected the sigmoid function neural network architectures from the three types of neural network architectures such as the sigmoid function neural network, the radial basis function (RBF) neural network and the polynomial neural network architecture. The brain and blood vessel regions are recognized and extracted accurately using the sigmoid function neural network architectures. In this study, the deep logistic GMDH-type neural network algorithm is applied to the medical image analysis of brain X-ray CT images and the skull and the blood vessel regions in the brain are recognized and extracted. The recognition results are compared with those obtained using the conventional neural networks trained 
using the back propagation algorithm and it is shown that this deep neural network is accurate and useful for the medical image analysis of the brain X-ray CT image.

\section{Deep GMDH-type neural network ${ }^{1}$}

The GMDH-type neural network architecture is shown in Fig.1. Here, nonlinear function $g_{i}$ is described by the following Kolmogorov-Gabor polynomial:

$$
g_{i}\left(x_{1}, x_{2}, \cdots, x_{p}\right)=a_{0}+\sum_{i} a_{i} x_{i}+\sum_{i} \sum_{j} a_{i j} x_{i} x_{j}+\cdots
$$

This nonlinear function is automatically organized by using the polynomial neurons. The architecture of the GMDH-type neural network is automatically organized using the heuristic self-organization and is produced as follows:

In the GMDH-type neural network, the original data are not separated into training and test sets because $\mathrm{AIC}^{7}$ or $\mathrm{PSS}^{8}$ can be used as the test errors.

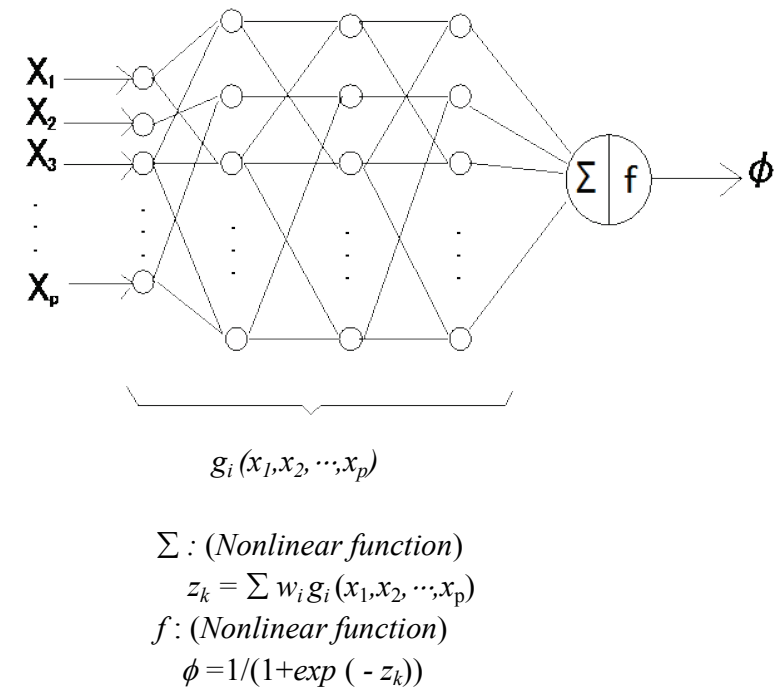

Fig.1 Architecture of the deep GMDH-type neural network

\subsection{The first layer}

$$
u_{j}=x_{j} \quad(j=1,2, \cdots, p)
$$

where $x_{\mathrm{j}}(\mathrm{j}=1,2, \cdots, p)$ are the input variables of the system, and $p$ is the number of input variables.

\subsection{The second layer}

All combinations of two variables $\left(u_{i}, u_{j}\right)$ are generated. For each combination, the neuron architecture is described by the following equations: $\sum:$ (Nonlinear function)

$$
z_{k}=w_{1} u_{i}+w_{2} u_{j}+w_{3} u_{i} u_{j}+w_{4} u_{i}^{2}+w_{5} u_{j}^{2}-w_{0} \theta_{1}
$$

$f:$ (Linear function)

$$
y_{k}=z_{k}
$$

where $\theta_{1}=1$ and $w_{i}(i=0,1,2, \cdots, 5)$ are weights between the first and second layer. The weights $w_{i}(i=0,1,2, \cdots, 5)$ are estimated by using the principal componentregression analysis. This procedure is as follows:

First, the values of $z_{k}$ are calculated using the following equation:

$$
z_{k}=\log _{e}\left(\phi^{\prime} /\left(1-\phi^{\prime}\right)\right)
$$

where $\phi^{\prime}$ is the normalized output variable. Then the weights $w_{i}(i=0,1,2, \cdots, 5)$ are estimated by using the principal component-regression analysis.

\section{[Principal component-regression analysis]}

In the GMDH-type neural network, the multicolinearity is generated in the function $\Sigma$ of the neurons because heuristic self-organization method is used. In this study, the function $\Sigma$ is calculated using the principal component-regression analysis.

In the case of Eq.(3), orthogonal vector $\underline{\boldsymbol{y}}$ is calculated .

Here,

$$
\underline{\boldsymbol{v}}=C \cdot \underline{\boldsymbol{u}}
$$

$$
\begin{aligned}
& \underline{\boldsymbol{v}}=\left(v_{1}, v_{2}, \ldots, v_{5}\right) \\
& \underline{\boldsymbol{u}}=\left(u_{i} u_{j}, u_{i} u_{j}, u_{i}^{2}, u_{j}^{2}\right)
\end{aligned}
$$

$\underline{\boldsymbol{v}}$ is orthonormal vectors and $C$ is orthonormal matrix. $C$ is calculated using the following eigenvalue equation.

$$
R \cdot C=C \cdot \Lambda
$$

Here, $R$ is a correlation matrix. Then, variable $z_{k}$ is calculated using orthogonal regression analysis.

$$
\begin{aligned}
z_{k} & =\underline{\boldsymbol{w}}^{\mathbf{T}} \cdot \underline{\boldsymbol{v}} \\
& =w_{1} v_{1}+w_{2} v_{2}+\ldots+w_{5} v_{5}
\end{aligned}
$$

Using the principal component-regression analysis, variable $z_{k}$ in the function $\Sigma$ is calculated without multicolinearity. In (8), useful orthogonal variables $v_{i}$ $(i=1,2, \ldots, 5)$ are selected by stepwise regression analysis ${ }^{9}$ using AIC or PSS criterion.

From these generated neurons, $L$ neurons which minimize $\mathrm{AIC}^{7}$ or $\mathrm{PSS}^{8}$ values are selected. The output values $\left(y_{k}\right)$ of $L$ selected neurons are set to the input values of the neurons in the third layer.

\subsection{The third and succeeding layers}

In the third and succeeding layers, the same computation of the second layer is iterated until AIC or 
PSS values of $L$ neurons are not decreased. When the iterative computation is terminated, the following calculation of the output layer is carried out.

\subsection{The output layer}

In the output layer, the output values of the neural network are calculated from $z_{k}$ as follows:

$$
y_{k}=1 /\left(1+\exp \left(-z_{k}\right)\right)
$$

So, in the output layer, the neuron architecture becomes as follows:

$\sum$ : (Nonlinear function)

$$
\begin{aligned}
& f: \text { (Nonlinear function) } \\
& \qquad \phi=1 /\left(1+\exp \left(-z_{k}\right)\right)
\end{aligned}
$$$$
z_{k}=\sum w_{i} g_{i}\left(x_{1}, x_{2}, \cdots, x_{p}\right)
$$

The complete neural network architecture is produced by selected neurons in each layer.

In this algorithm, the principal componentregression analysis is used for the learning calculation of the neural network and the accurate and stable prediction values are obtained. The structural parameters such as the number of layers, the number of neurons in hidden layers and useful input variables are automatically selected to minimize prediction error criterion defined as AIC or PSS. The GMDH-type neural network is organized with two types of neurons which are the sigmoid function neuron and the polynomial neuron and this neural network can identify very complex nonlinear system.

\section{Application to the medical image analysis of brain X-ray CT images.}

In this study, the regions of the skull and the blood vessels in the brain were recognized and extracted automatically. Multi-detector row CT (MDCT) images of the brain are used in this study.

\subsection{Extraction of the skull regions}

An brain MDCT image shown in Fig.2 was used for organizing the deep GMDH-type neural network. The statistics of the image densities and $x$ and $y$ coordinates in the neighboring regions, the $N \times N$ pixel regions, were used as the image features. The neural networks were organized when the values of $N$ were from 3 to 10 . When $N$ was equal to 3 , the neural network architecture had the smallest recognition error. Fig. 3 shows the variation of PSS values in each layer. The PSS values were decreased gradually through the layers and small PSS value was obtained in the seventh layer. The deep GMDH-type neural network output the skull image (Fig.4) and the post-processing analysis of the output skull image was carried out. Fig.5 shows the output image after the post-processing. The output image after the post-processing was overlapped to the original image (Fig.2) as shown in Fig.6. The recognized skull region are very accurate. The skull region was extracted from the original image as shown in Fig.7. These image processing were carried out for all slices by the organized deep GMDH-type neural network and the 3dimensional skull image was generated as shown in Fig.8. A conventional neural network trained using the back propagation algorithm was applied to the same recognition problem. The output images, when the numbers of neurons in the hidden layer $(m)$ are 5,7 and 9, are shown in Fig.9. These images contain more regions which are not part of the skull.

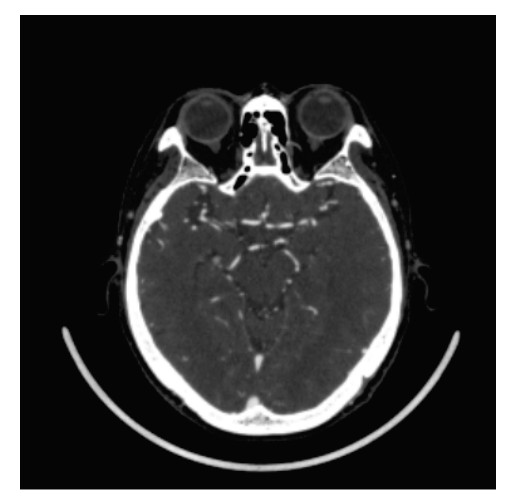

Fig. 2 Original image of the brain

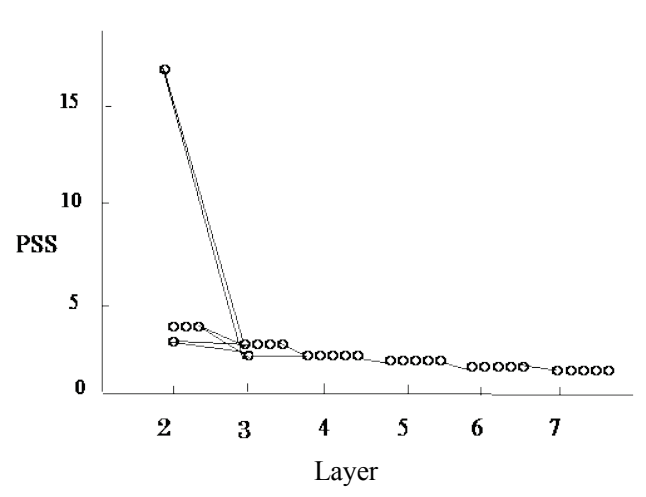

Fig. 3 Variation of PSS values in each layer (1) 


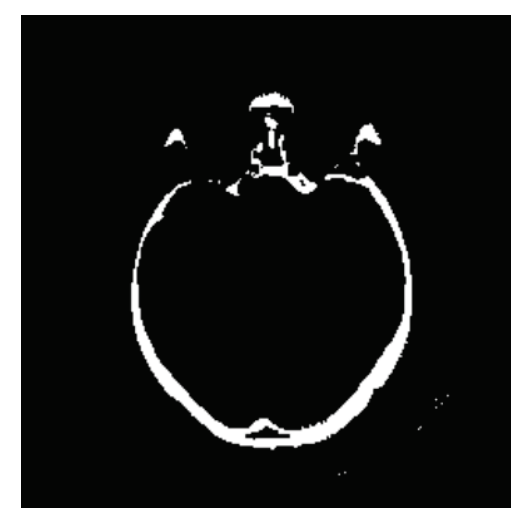

Fig. 4 Output image (1)

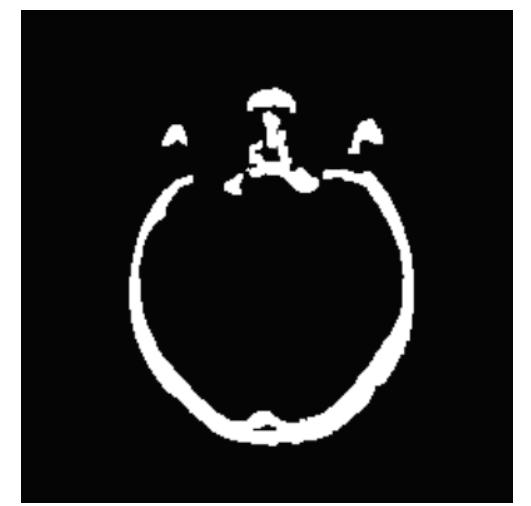

Fig. 5 Output image after the post processing (1)

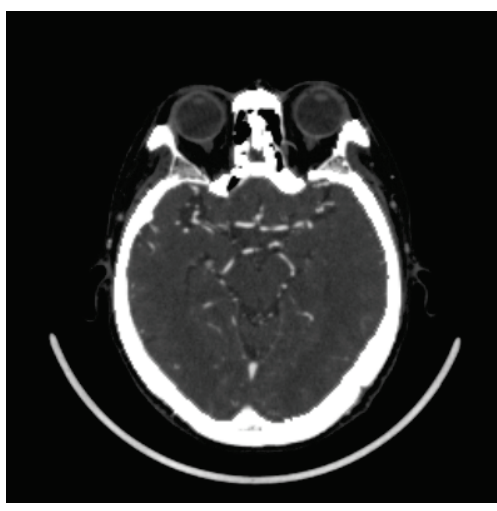

Fig.6 Overlapped image (1)

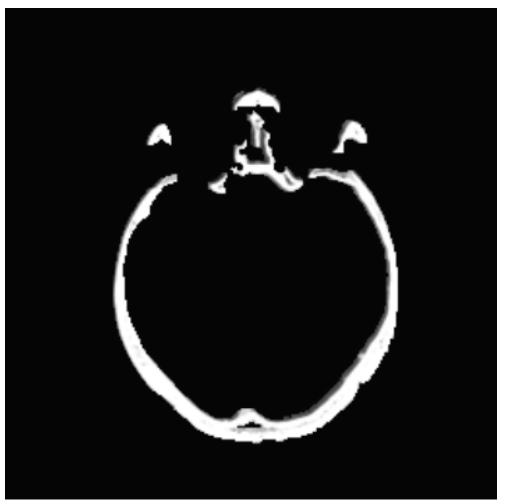

Fig.7 Extracted gray scale image (1) of the skull

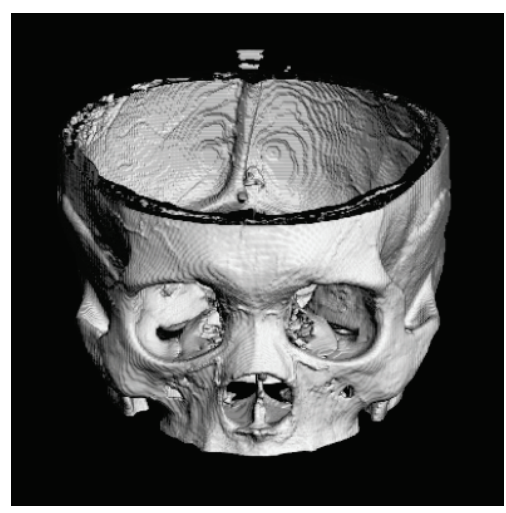

Fig.8 The 3-dimensional skull image

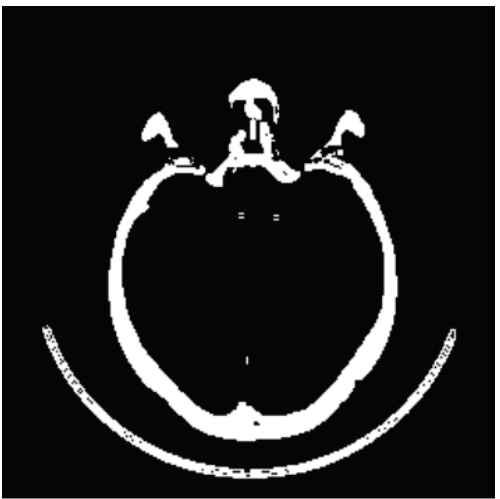

(a) $m=5$

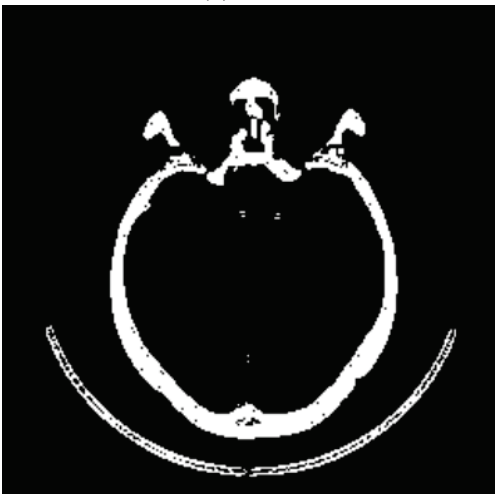

(b) $m=7$

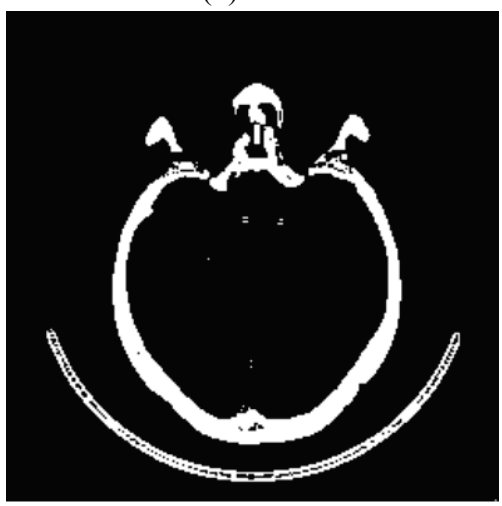

(c) $m=9$

Fig.9 Output images of the conventional neural network (1) 


\subsection{Extraction of the blood vessel regions}

Another deep GMDH-type neural network was organized and applied to the recognition of the blood vessel regions in the brain using the MDCT image shown in Fig.10 which was extracted from the original image in Fig.2. Fig.11 shows the variation of PSS values in each layer. Very small PSS values were obtained in the seventh layer. The deep GMDH-type neural network output the blood vessel image as shown in Fig.12. The output image after the post-processing (Fig.13) was overlapped as shown in Fig.14. The recognized blood vessel regions were very accurate. The blood vessel regions were extracted from the original image as shown in Fig. 15. These image processing were carried out for all slices by the organized deep GMDHtype neural network and the 3-dimensional blood vessel image was generated as shown in Fig.16. A conventional neural network trained using the back propagation algorithm was applied and the output images, when the numbers of neurons in the hidden layer $(m)$ are 5, 7 and 9, are shown in Fig.17. These images contained many noise compared with the output image of the deep GMDH-type neural network which is shown in Fig. 12.

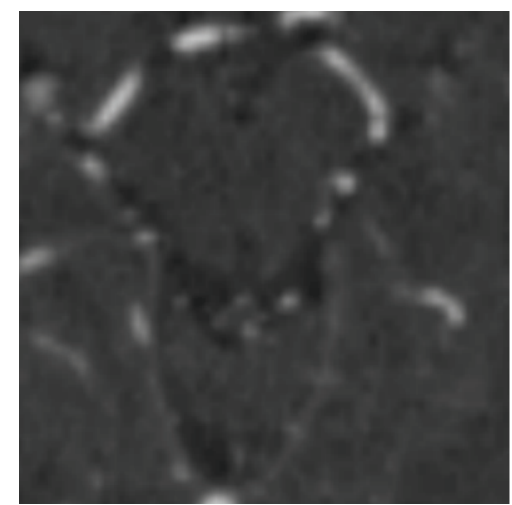

Fig.10 Original image of blood vessels extracted from Fig.2

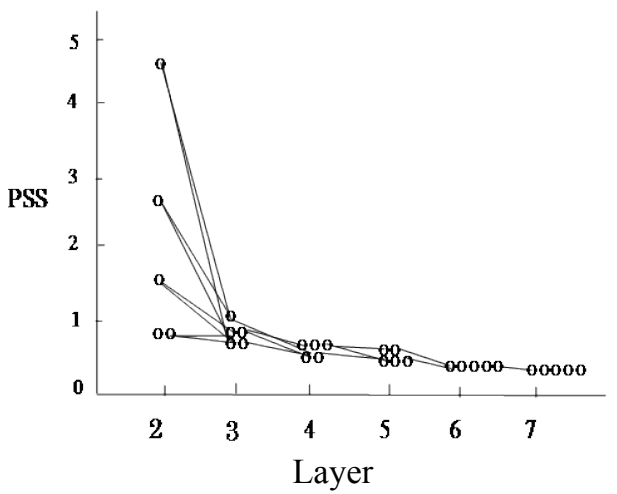

Fig. 11 Variation of PSS values in each layer (2)

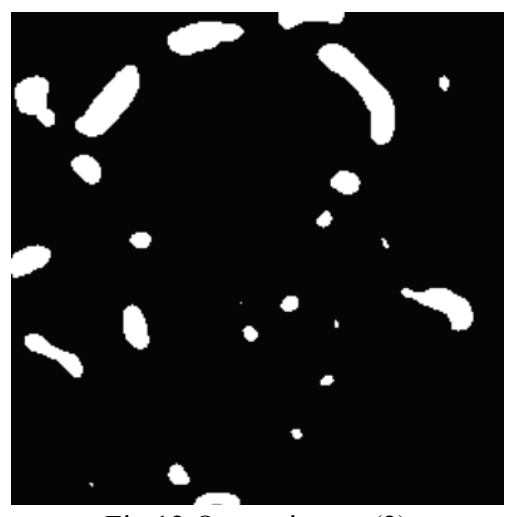

Fig.12 Output image (2)

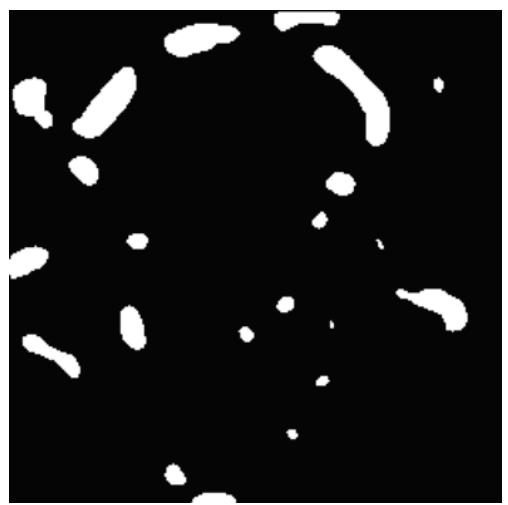

Fig.13 Output image after the post-processing (2)

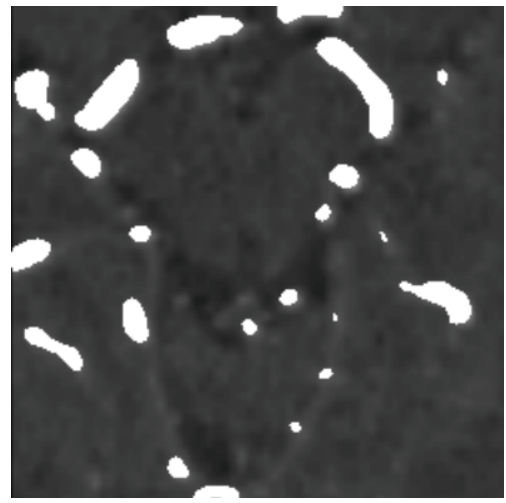

Fig.14 Overlapped image (2)

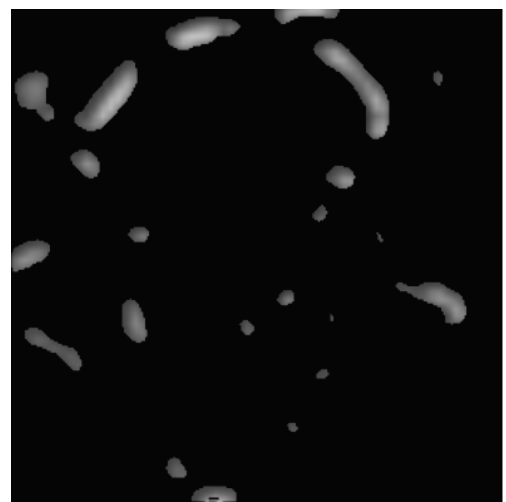

Fig.15 Extracted gray scale image (2) of blood vessels 


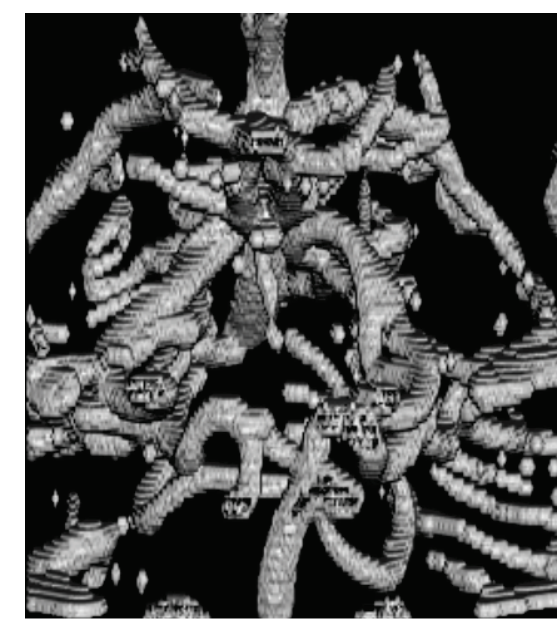

Fig.16 The 3-dimensional blood vessel image

\section{Conclusions}

In this paper, the deep GMDH-type neural network algorithm using principal component-regression analysis was applied to the medical image analysis of the brain X-ray CT image, and the skull and the blood vessel regions in the brain were recognized and extracted accurately. The deep neural network architectures which had six hidden layers were organized for both skull and blood vessel regions so as to minimize the prediction error criterion defined as PSS. PSS values were gradually decreased through the hidden layers and these PSS values converged on very small PSS value in the seventh layer. The recognition results were compared with those obtained using the conventional neural networks trained using the back propagation algorithm and it was shown that this deep neural networks with many hidden layers were accurate and useful for the medical image analysis of the brain $\mathrm{X}$-ray CT image.

\section{Acknowledgment}

This work was supported by (JSPS) KAKENHI 26420421 .

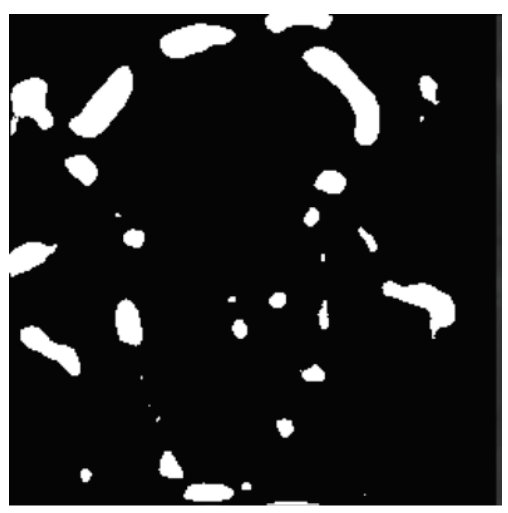

(a) $m=5$

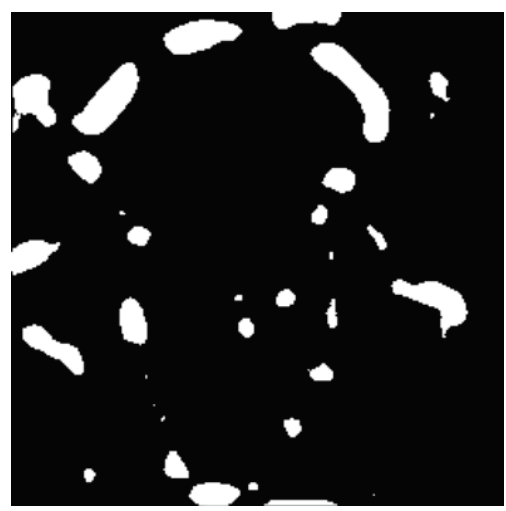

(b) $m=7$

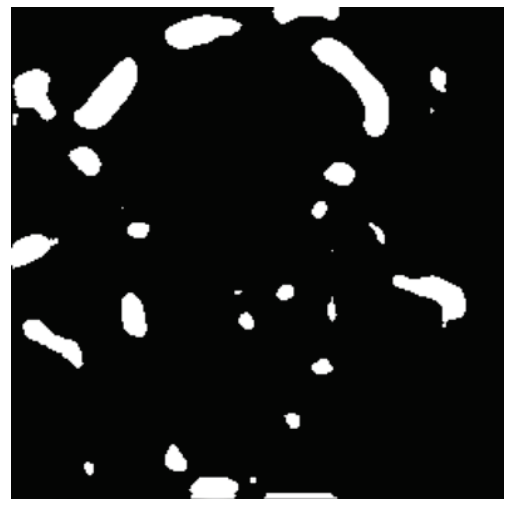

(c) $m=9$

Fig.17 Output images of the conventional neural network (2) 


\section{References}

1. T. Kondo, J. Ueno and S. Takao, Logistic GMDH-type neural network using principal component-regression analysis and its application to medical image diagnosis of lung cancer, Artificial Life and Robotics 20(2)(2015) 137144.

2. T. Kondo, J. Ueno and S. Takao, Deep multi-layered GMDH-type neural network using principal componentregression analysis and its application to medical image recognition of brain and blood vessels, Proceedings of the twentieth international symposium on artificial life and robotics (2015) pp.92-95.

3. T. Kondo, J. Ueno and S. Takao, Medical image recognition of heart regions by deep multi-layered GMDH-type neural network using principal componentregression analysis, Journal of Robotics, Networking and Artificial Life, 2(3)(2015) 166-172.

4. T. Kondo, J. Ueno and S. Takao, Deep feedback GMDHtype neural network using principal component- regression analysis and its application to medical image recognition of abdominal multi-organs, Journal of Robotics, Networking and Artificial Life 2(2)(2015) 9499.

5. A. G. Ivakhnenko, Heuristic self-organization in problems of engineering cybernetics, Automatica 6(2)(1970) 207-219.

6. S. J. Farlow ed., Self-organizing methods in modeling, GMDH-type algorithm, New York: Marcel Dekker Inc., 1984.

7. H. Akaike, A new look at the statistical model identification, IEEE Trans. Automatic Control, AC-19, (6) (1974) 716-723.

8. H. Tamura, T. Kondo, Heuristics free group method of data handling algorithm of generating optimum partial polynomials with application to air pollution prediction, Int. J. System Sci. 11 (9) (1980) 1095-1111.

9. N. R. Draper and H. Smith, Applied Regression Analysis, New York: John Wiley and Sons, 1982. 